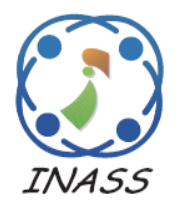

\title{
Link Balance and Performance of UAM in NOMA-based Cellular Networks
}

\author{
Nam-Soo Kim ${ }^{1 *}$ \\ ${ }^{1}$ Department of Electronic Engineering, Cheongju University, Republic of Korea \\ * Corresponding author's Email: nskim@cju.ac.kr
}

\begin{abstract}
Recently, the non-orthogonal multiple access (NOMA) technique, which transmits multiple users in the same time-frequency block, has been focused on for future cellular networks. Conventional NOMA-based aerial cellular networks consider an unmanned aerial vehicle (UAV) as an aerial user, in which secure control information for UAV is important. In recent days, the deployment of urban air mobility (UAM) has been actively discussed for a new aerial transport service in an urban area. Since UAM conveys passengers, both the traffic and the control information for passengers and a vehicle are important. Therefore, this paper considers a NOMA-based aerial cellular network with a UAM as an aerial user and a ground user as a terrestrial user. And we derive closed-form outage probabilities of the forward and reverse links of UAM, and the Monet-Carlo simulation verifies the results. Further, the condition to maintain the balance between the forward and the reverse links for the identical quality of service (QoS) is derived. Numerical results show that the outage probability of the forward link decreases with the increase of the received signal-to-noise ratio (SNR). However, we noticed that the outage probability of the reverse link shows the error floor, which does not decrease with the received SNR and remains constant, caused by the interference from the ground user $(\mathrm{GU})$. Also, it is shown that there is an average transmit power of UAM to conform the link balance regardless of strong or weak users.
\end{abstract}

Keywords: Cellular network, Link balance, NOMA, Link performance, UAM.

\section{Introduction}

Non-orthogonal multiple access (NOMA) for the future cellular networks was first proposed by $Y$. Saito et. al., which has higher spectral efficiency and lower latency than conventional orthogonal multiple access (OMA) [1]. These advantages draw much attention to applying the 5th generation cellular networks and beyond, and consequent studies have been actively reported; performance analysis of NOMA systems which transmit multiple users simultaneously [2, 3], optimum power allocation and power control for maximum capacity [4], cooperative NOMA system [5, 6], analysis of NOMA Multipleinput multiple-output (MIMO) system [7, 8], and application for cognitive NOMA systems [9-11].

Recently, NOMA-based aerial systems are considered as well as terrestrial NOMA systems. The outage performance of ground users (GU) with an aerial base station (BS) of unmanned aerial vehicle (UAV) was published [12, 13]. Also, the performance improvement of GU with UAV relay has been studied $[14,15]$. The maximum achievable rate of UAV with maintaining quality-of-service (QoS) through control link is derived [16]. More recently, an adaptive reinforcement learning approach is applied to maximize the sum-rate of an NOMA-based UAV system [17].

Nowadays, the deployment of urban air mobility (UAM), which transports cargo and passengers in a city, is actively considered [18]. Most of the conventional studies of NOMA-based aerial systems consider a UAV as an aerial user (AU). Generally, UAVs require highly reliable communication for a designated control link but a lower data rate. However, in the case of UAM, control information for an unmanned vehicle and traffic information for passengers have to be transferred with the predetermined QoS. The QoS balance must be kept 
between the forward and reverse links, especially for the passenger's voice traffic. For example, when a significant quality difference between the links exist, the speaker doesn't feel comfortable during voice communication.

Therefore, this study considers a UAM NOMA cellular system, consisting of a terrestrial BS, a terrestrial user (GU), and UAM as an aerial user (AU) in a cell. And we derive the performance of the forward link and reverse link of UAM in closed-form. Also, the condition and the average transmit power of UAM for the link balance are derived. A summary of the contribution is as follows:

- derive closed-form expressions of the forward and reverse link outage probabilities of UAM under strong and weak user conditions.

- derive the probabilities that the UAM becomes the weak or strong users in closed-form.

- derive the average outage probabilities of forward and reverse links of UAM

- derive power allocation ratio and average transmit power of UAM for link balance

The rest of this paper is organized as follows. Section II presents a UAM NOMA system and channel models. The outage probabilities of the forward and reverse links for UAM are described in section III. Also, the power allocation coefficient and transmit power of UAM to link balance are derived in section IV. Section V shows the numerical examples of outage probability and compares the analytical and the Monte-Carlo simulation results. It is shown that the results are exactly matched. Finally, the conclusions of this study are given and future research subject is addressed in section $\mathrm{V}$.

\section{System model}

Fig. 1 shows the NOMA-based aerial system model, which consists of a BS, a GU, and an AU. Most of the terrestrial cellular systems use the terminology of a downlink to GU and uplink to BS.

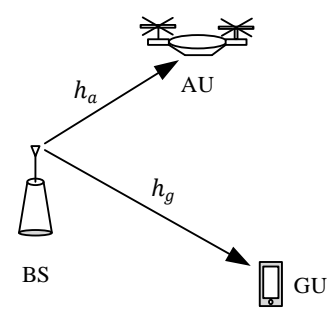

(a)

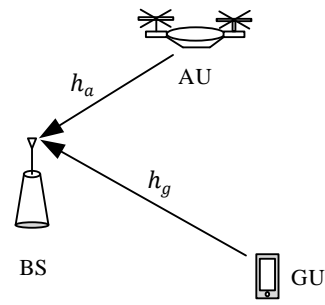

(b)
Figure. 1 NOMA-based UAM system model: (a) forward link and (b) reverse link
However, these terminologies confuse aerial systems. Here we use forward link and reverse link instead of downlink and uplink, respectively.

\subsection{Channel model}

BS-GU link is a terrestrial link of which channel coefficients denotes $h_{g}$ in Fig. 1. Generally, it is assumed that several indirect paths exist without a direct path in a metropolitan city where UAM is introduced. Hence the channel coefficient $h_{g}$ has complex Gaussian distribution with unit variance and zero mean, $h_{g} \sim \mathrm{CN}(0,1)$. For the notational convenience denote $H_{g}=\left|h_{g}\right|^{2}$, then the probability density function (pdf) of $H_{g}$ can be written by

$$
f_{H_{g}}(x)=\frac{1}{\Omega_{g}} e^{-x / \Omega_{g}}
$$

where $\Omega_{g}$ is the mean-square value of $H_{g}$, we assume $\Omega_{g}=1$. Also, the cumulative distribution function $(\mathrm{CDF})$ is given by

$$
F_{H_{g}}(x)=1-e^{-x} .
$$

The channel gain of BS-GU link is denoted

$$
G_{g}=\frac{1}{A d_{g}^{\alpha g}}
$$

where $\mathrm{A}=\left(4 \pi f_{c} / c\right)^{2}, f_{c}$ is the carrier frequency, and $c$ is the speed of light [16]. And $\alpha_{g}$ is the ground path-loss exponent with $\alpha_{g}=\alpha_{e}+\alpha_{0}$ where $\alpha_{e}$ and $\alpha_{0}$ are values based on the line-of-sight (LOS) or non-line of sight (NLOS) link [12], [15]. $d_{g}$ denotes the distance between the BS and the GU.

While BS-AU link is an aerial link of which channel coefficient denotes $h_{a}$ in Fig. 1. Generally, it is assumed that there exist both indirect and direct paths in the aerial link. Therefore the pdf of $h_{a}$ is generally assumed to be Rician pdf. Similarly, denote $H_{a}=\left|h_{a}\right|^{2}$, then the pdf of $H_{a}$ can be written by [19]

$$
\begin{aligned}
& f_{H_{a}}(x)= \\
& \frac{(1+K) e^{-K}}{\Omega_{a}} \exp \left[-\frac{(1+K) x}{\Omega_{a}}\right] I_{0}\left(2 \sqrt{\frac{K(1+K) x}{\Omega_{a}}}\right)
\end{aligned}
$$

where $\Omega_{a}$ is the mean-square value of $H_{a}$. We assume $\Omega_{a}=1 . K$ denotes the Rician factor which is the ratio of the direct path power-to-indirect path power. When the altitude angle between a BS and UAM is greater, the LOS power which is a direct path is stronger and the NLOS power which is an indirect 
path is weaker. The relationship between the Rician factor and the altitude angle of $\theta$ [rad] is given by [15-16]

$$
K=K_{\min } \exp \left\{\frac{2}{\pi} \ln \left(\frac{K_{\max }}{K_{\min }} \theta\right)\right\}
$$

where $K_{\min }$ and $K_{\max }$ are Rician factors for $\theta=0$ and $\theta=\pi / 2$, respectively. And $I_{0}(\cdot)$ denotes the zeroth-order modified Bessel function of the first kind $[22,8.445]$. The CDF becomes

$$
F_{H_{a}}(x)=1-Q(\sqrt{2 K}, \sqrt{2(1+K) x})
$$

where $Q(a, b)$ is the Marcum $\mathrm{Q}$ function, $Q(a, b)=$ $\int_{b}^{\infty} x e^{-\frac{a^{2}+x^{2}}{2}} I_{0}(a x) d x$. Similarly, the channel gain of the BS-AU link is given by [12], [15]

$$
G_{a}=\frac{1}{A d_{a}^{\alpha_{a}}}
$$

where $\alpha_{a}$ denotes the aerial path-loss exponent, $\alpha_{a}=\alpha_{e}\left(1-P_{L O S}\right)+\alpha_{0}$ where $\alpha_{e}$ and $\alpha_{0}$ are constant depending on the path loss coefficient. $P_{L O S}$ is the probability of LOS [20]. $d_{a}$ denotes the distance between the $\mathrm{BS}$ and the $\mathrm{AU}$.

\subsection{Forward link}

According to the NOMA protocol, BS transmits the superposed signals with the information to $\mathrm{AU}$ and GU, $\sqrt{\alpha_{s} P_{b}} x_{s}+\sqrt{\alpha_{w} P_{b}} x_{w}$. Where $P_{b}$ denotes the transmit power from BS, and $\alpha_{s}$ and $\alpha_{w}$ are the power allocation coefficients to a strong user and a weak user, respectively, $\alpha_{s} \leq \alpha_{w}$ with $\alpha_{s}+\alpha_{w}=1$. $x_{s}$ and $x_{w}$ are bipolar messages of a strong user and a weak user, respectively, with the ensemble average of $\mathrm{E}\left[\left|x_{s}\right|^{2}\right]=\mathrm{E}\left[\left|x_{w}\right|^{2}\right]=1$. In the case of an AU, the received signal strength from a $B S$ fluctuates with the height, intensity, and arrangement of the buildings along the aerial path. In the case of the ensure information such as control information for the unmanned flights, we can allocate more power to AU than GU.

The received signal of AU via the forward link can be written by

$$
y_{a}=h_{a}\left(\sqrt{\alpha_{s} P_{b} G_{s}} x_{s}+\sqrt{\alpha_{w} P_{b} G_{w}} x_{w}\right)+n_{a}
$$

where $G_{s}$ and $G_{w}$ denote channel gains of the BSstrong user and BS-weak user, respectively. $n_{a}$ represents white Gaussian noise with zero mean and variance of $N_{0}, n_{a} \sim C N\left(0, N_{0}\right)$. In NOMA system, the decision of the strong or weak user is determined by the magnitude of the instantaneous channel gain. By considering the signal component from the strong user as noise, the weak user decodes its information from the received signal. During the decoding process of the strong user, the component from the weak user is removed by the successive interference cancellation (SIC). After SIC, the strong user decodes. Therefore, the signal-to-interference plus noise (SINR) of AU through the forward link can be written by [5]

$$
\gamma_{a}^{F}=\left\{\begin{array}{l}
\frac{\left|h_{a}\right|^{2} \alpha_{w} K_{1} \rho_{a}}{\left|h_{a}\right|^{2} \alpha_{s} K_{1} \rho_{a}+1},\left|h_{a}\right|^{2} G_{a} \leq\left|h_{g}\right|^{2} G_{g} \\
\left|h_{a}\right|^{2} \alpha_{s} K_{1} \rho_{a}, \quad\left|h_{a}\right|^{2} G_{a}>\left|h_{g}\right|^{2} G_{g}
\end{array}\right.
$$

where $\rho_{a}$ denotes the signal-to-noise ratio (SNR) of $\mathrm{AU}, \rho_{a}=P_{a} G_{a} / N_{0} . K_{1}$ is the power ratio of transmit power of BS to transmit power of $\mathrm{AU}, K_{1}=P_{b} / P_{a}$. Notice the performance of the forward link is influenced by the power allocation coefficient in Eq. (9).

The capacity of AU in a forward link can be obtained from Eq. (9)

$$
R_{a}^{F}=\log \left(1+\gamma_{a}^{F}\right) .
$$

\subsection{Reverse link}

Different from the forward link, users in a cell transmit the signal through independent channels separately in the reverse link. The transmit signals from AU and GU are $\sqrt{P_{a}} x_{a}$ and $\sqrt{P_{g}} x_{g}$, respectively. Where $P_{a}$ and $P_{g}$ are transmitting powers and $x_{a}$ and $x_{g}$ are information of $\mathrm{AU}$ and $\mathrm{GU}$, respectively. We assume each channel is reciprocal.

The received signal at $\mathrm{BS}$ from $\mathrm{GU}$ and $\mathrm{AU}$ through the reverse link can be written by

$$
y_{b}=h_{a} \sqrt{P_{a} G_{a}} x_{a}+h_{g} \sqrt{P_{g} G_{g}} x_{g}+n_{b}
$$

where $G_{a}$ and $G_{g}$ denote the channel gain of BS-AU and BS-GU, respectively. $n_{b}$ is white Gaussian noise with zero mean and variance of $N_{0}, n_{b} \sim C N\left(0, N_{0}\right)$. The received SINR, $\gamma_{b}^{R}$, at $\mathrm{BS}$ from $\mathrm{AU}$ is given by

$$
\gamma_{b}^{R}= \begin{cases}\frac{\left|h_{a}\right|^{2} \rho_{a}}{\left|h_{g}\right|^{2} \rho_{g}+1}, & \left|h_{a}\right|^{2} G_{a} \geq\left|h_{g}\right|^{2} G_{g} \\ \left|h_{a}\right|^{2} \rho_{a}, & \left|h_{a}\right|^{2} G_{a}<\left|h_{g}\right|^{2} G_{g}\end{cases}
$$


where $\rho_{g}$ denotes received SNR from GU, $\rho_{g}=$ $P_{g} G_{g} / N_{0}$. Different from Eq. (9), note that the performance of the reverse link is affected by the interference from GU.

Similarly, the capacity of AU via a reverse link can be written by

$$
R_{a}^{R}=\log \left(1+\gamma_{b}^{R}\right)
$$

\section{Outage probability}

In this section, we drive the probabilities of a weak user and a strong user with the given channel condition and the outage probabilities of UAM in forward and reverse links. And the conditions for link balances are addressed.

\subsection{Probability of strong/weak user}

According to the instantaneous received signal strength, an UAM can be a strong or weak user. The probability of a weak user of UAM can be written by

$$
\begin{aligned}
\operatorname{Pr} & \left(\left|h_{a}\right|^{2} G_{a} \leq\left|h_{g}\right|^{2} G_{g}\right)=\operatorname{Pr}\left(H_{a}<H_{g} \frac{G_{g}}{G_{a}}\right) \\
& =1-\underbrace{\int_{0}^{\infty} Q\left(\sqrt{2 K}, \sqrt{2(1+K) \frac{G_{g}}{G_{a}} \chi}\right) e^{-\chi} d \chi}_{\Phi_{1}}(14)
\end{aligned}
$$

where $Q(a, b)$ is given by [21]

$$
Q(a, b)=e^{-a^{2} / 2} \sum_{k=0}^{\infty}\left(\frac{1}{k !}\right)^{2}\left(\frac{a^{2}}{2}\right)^{k} \Gamma\left(1+k, \frac{b^{2}}{2}\right)
$$

and where $[22,8.352 .2]$

$$
\Gamma(1+n, x)=n ! e^{-x} \sum_{m=0}^{n} \frac{x^{m}}{m !} .
$$

$\Phi_{1}$ in Eq. (14) can be arranged by Eq. (15) and Eq. (16)

$$
\begin{aligned}
& \Phi_{1}=e^{-K} \sum_{k=0}^{\infty} \frac{1}{k !} K^{k} \\
& \sum_{m=0}^{K} \frac{(1+K)^{m}}{m !}\left(\frac{G_{g}}{G_{a}}\right)^{m} \underbrace{\int_{0}^{\infty} e^{-\left\{(1+K) \frac{G_{g}}{G_{a}}+1\right\} \chi} \chi^{m} d \chi}_{\Phi_{2}}
\end{aligned}
$$

By applying $[22,3.351 .3]$

$$
\int_{0}^{\infty} x^{n} e^{-\mu x} d x=n ! \mu^{-n-1},
$$

$\Phi_{2}$ can be written by

$$
\Phi_{2}=m !\left\{(1+K) \frac{G_{g}}{G_{a}}+1\right\}^{-m-1} .
$$

Replacing Eq. (17) and Eq. (19) into Eq. (14), we have

$$
\begin{aligned}
& \operatorname{Pr}\left(\left|h_{a}\right|^{2} G_{a} \leq\left|h_{g}\right|^{2} G_{g}\right) \\
& =1-e^{-K} \sum_{k=0}^{\infty} \frac{1}{k !} K^{k} \sum_{m=0}^{k} \frac{(1+K)^{m} G_{a}}{G_{g}\left(1+K+G_{a} / G_{g}\right)^{m+1}} .
\end{aligned}
$$

Also, the probability of strong user can be obtained from Eq. (20),

$$
\begin{aligned}
& \operatorname{Pr}\left(\left|h_{a}\right|^{2} G_{a}>\left|h_{g}\right|^{2} G_{g}\right) \\
& \quad=1-\operatorname{Pr}\left(\left|h_{a}\right|^{2} G_{a} \leq\left|h_{g}\right|^{2} G_{g}\right) .
\end{aligned}
$$

\subsection{Forward link outage probability}

An outage happens when the capacity is less than the predefined threshold rate. The outage probability of UAM in the forward link can be written by

$$
P_{o, F}=\operatorname{Pr}\left(R_{a}^{F}<R\right)=\operatorname{Pr}\left(\gamma_{a}^{F}<\Gamma_{a}\right)
$$

where $R[\mathrm{bit} / \mathrm{Hz}]$ is the predefined threshold rate and the second equality is applied Eq. (15) with the threshold of $\Gamma_{a}=2^{R}-1$. It means that the received SINR exceeds $\Gamma_{a}$, then the system capacity rate is higher than $R$. Hence $\Gamma_{a}$ denotes the SINR that satisfies minimum QoS. Replacing Eq. (13) into Eq. (22), we can write

$$
P_{o, F}=\operatorname{Pr}\left(H_{a}<\phi\right)=1-Q(\sqrt{2 K}, \sqrt{2(1+K) \phi})
$$

where

$$
\phi=\left\{\begin{array}{l}
\frac{\Gamma_{a}}{K_{1} \rho_{a}\left(\alpha_{w}-\Gamma_{a} \alpha_{s}\right)},\left|h_{a}\right|^{2} G_{a} \leq\left|h_{g}\right|^{2} G_{g} \\
\frac{\Gamma_{a}}{\alpha_{s} K_{1} \rho_{a}}, \quad\left|h_{a}\right|^{2} G_{a}>\left|h_{g}\right|^{2} G_{g}
\end{array} .\right.
$$

From Eq. (15) and Eq. (16), Eq. (23) can be rearranged by 


$$
\begin{aligned}
& P_{o, F} \\
& =1-e^{-\{K+(K+1) \phi\}} \sum_{k=0}^{\infty} \frac{1}{k !} K^{k} \sum_{m=0}^{k} \frac{\{(K+1) \phi\}^{m}}{m !} .
\end{aligned}
$$

Since $\phi$, which is given in Eq. (24), depends on the channel condition, Eq. (25) can be decomposed by

$$
\begin{aligned}
P_{o, F}= & \operatorname{Pr}\left(H_{a}<\left.\phi|| h_{a}\right|^{2} \leq\left|h_{g}\right|^{2} G_{g}\right) \\
& \times \operatorname{Pr}\left(\left|h_{a}\right|^{2} G_{a} \leq\left|h_{g}\right|^{2} G_{g}\right) \\
& +\operatorname{Pr}\left(H_{a}<\left.\phi|| h_{a}\right|^{2} G_{a}>\left|h_{g}\right|^{2} G_{g}\right) \\
& \times \operatorname{Pr}\left(\left|h_{a}\right|^{2} G_{a}>\left|h_{g}\right|^{2} G_{g}\right)
\end{aligned}
$$

where the first conditional probability is the probability that UAM is the weak user. By replacing Eq. (24) into Eq. (25), the first conditional probability becomes

$$
\begin{aligned}
& \operatorname{Pr}\left(H_{a}<\left.\phi|| h_{a}\right|^{2} G_{a} \leq\left|h_{g}\right|^{2} G_{g}\right) \\
& =1 \\
& -e^{-\left\{K+\frac{(K+1) \Gamma_{a}}{K_{1} \rho_{a}\left(\alpha_{w}-\Gamma_{a} \alpha_{s}\right)}\right\}} \sum_{k=0}^{\infty} \frac{1}{k !} K^{k} \sum_{m=0}^{k} \frac{\left\{\frac{(K+1) \Gamma_{a}}{K_{1} \rho_{a}\left(\alpha_{w}-\Gamma_{a} \alpha_{s}\right)}\right\}^{m}}{m !} .
\end{aligned}
$$

Similarly, the third conditional probability is the probability that UAM is strong user, we have

$$
\begin{aligned}
& \operatorname{Pr}\left(H_{a}<\left.\phi|| h_{a}\right|^{2} G_{a}>\left|h_{g}\right|^{2} G_{g}\right)= \\
& \quad e^{-\left\{K+\frac{(K+1) \Gamma a}{\alpha_{S} K_{1} \rho_{a}}\right\}} \sum_{k=0}^{\infty} \frac{1}{k !} K^{k} \sum_{m=0}^{k} \frac{\left\{\frac{(K+1) \Gamma a}{\alpha_{S} K_{1} \rho_{a}}\right\}^{m}}{m !} .
\end{aligned}
$$

Consequently, the outage probability of UAM in the forward link can be obtained from Eq. (20), Eq. (21), Eq. (27), Eq. (28), and Eq. (26).

\subsection{Reverse link outage probability}

Similarly, the outage probability of UAM in the reverse link is determined that the capacity of $R_{a}^{R}$ in Eq. (13) is less than the threshold. The outage probability is given by

$$
P_{o, R}=\operatorname{Pr}\left(R_{a}^{R}<R\right)=\operatorname{Pr}\left(\gamma_{b}^{R}<\Gamma_{a}\right) .
$$

From Eq. (13), the outage probability in Eq. (29) becomes

$$
P_{o, R}=\operatorname{Pr}\left(H_{a}<\varphi\right)=1-\mathrm{Q}(\sqrt{2 K}, \sqrt{2(1+K) \varphi})
$$

where

$$
\varphi=\left\{\begin{array}{ll}
\frac{\Gamma_{a}}{\rho_{a}}, & \left|h_{a}\right|^{2} G_{a} \leq\left|h_{g}\right|^{2} G_{g} \\
\frac{\Gamma_{a}\left(H_{g} \rho_{g}+1\right)}{\rho_{a}}, & \left|h_{a}\right|^{2} G_{a}>\left|h_{g}\right|^{2} G_{g}
\end{array} .\right.
$$

According to the given condition, $\varphi$ has different values. Therefore, Eq. (30) can be written by

$$
\begin{aligned}
P_{o, R}= & P r \\
& \left(H_{a}<\left.\varphi|| h_{a}\right|^{2} G_{a} \leq\left|h_{g}\right|^{2} G_{g}\right) \\
& \times \operatorname{Pr}\left(\left|h_{a}\right|^{2} G_{a} \leq\left|h_{g}\right|^{2} G_{g}\right) \\
& +\operatorname{Pr}\left(H_{a}<\left.\varphi|| h_{a}\right|^{2} G_{a}>\left|h_{g}\right|^{2} G_{g}\right) \\
& \times \operatorname{Pr}\left(\left|h_{a}\right|^{2} G_{a}>\left|h_{g}\right|^{2} G_{g}\right),
\end{aligned}
$$

where the first conditional probability is the probability that UAM is the weak user. Replace $\varphi$ and $\phi$ in Eq. (27), the first conditional probability becomes

$$
\begin{aligned}
& \operatorname{Pr}\left(H_{a}<\left.\varphi|| h_{a}\right|^{2} G_{a} \leq\left|h_{g}\right|^{2} G_{g}\right) \\
= & 1 \\
- & e^{-\left\{K+\frac{(K+1) \Gamma_{a}}{\rho_{a}}\right\}} \sum_{k=0}^{\infty} \frac{1}{k !} K^{k} \sum_{m=0}^{k} \frac{\left\{\frac{(K+1) \Gamma_{a}}{\rho_{a}}\right\}^{m}}{m !}
\end{aligned}
$$

Similarly, the third conditional probability is the probability that UAM is a strong user, we have

$$
\begin{gathered}
\operatorname{Pr}\left(H_{a}<\left.\varphi|| h_{a}\right|^{2} G_{a}>\left|h_{g}\right|^{2} G_{g}\right)= \\
1-\underbrace{\int_{0}^{\infty} Q\left(\sqrt{2 K}, \sqrt{\frac{2(1+K) \Gamma_{a}\left(\chi \rho_{g}+1\right)}{\rho_{a}}}\right) e^{-\chi} d \chi}_{\Psi_{1}}
\end{gathered}
$$

where $\Psi_{1}$ denotes an average of the random variable of $H_{g}$. Applying Eq. (15) and Eq. (16), $\Psi_{1}$ can be rearranged by

$\Psi_{1}$

$=e^{-K} \sum_{k=0}^{\infty} \frac{1}{k !} K^{k} e^{-\frac{(1+K) \Gamma a}{\rho_{a}}} \sum_{m=0}^{k} \frac{(1+K)^{m}}{m !}\left(\frac{\Gamma_{a} \rho_{g}}{\rho_{a}}\right)^{m} \Psi_{2}$

where

$$
\Psi_{2}=\int_{0}^{\infty} e^{-\left\{\frac{(1+K) \Gamma_{a} \rho_{g}}{\rho_{a}}+1\right\} \chi}\left(\chi+\frac{1}{\Gamma_{a} \rho_{g}}\right)^{m} d \chi
$$




$$
\begin{gathered}
=\left\{\frac{(1+K) \Gamma_{a} \rho_{g}}{\rho_{a}}+1\right\}^{-m-1} \exp \left(\frac{(1+K) \Gamma_{a}}{\rho_{a}}\right. \\
\left.+\frac{1}{\rho_{g}}\right) \Psi_{3} .
\end{gathered}
$$

The second equality is applied to $[22,3.382 .3]$

$$
\begin{aligned}
\int_{0}^{\infty} & (x+\beta)^{v} e^{-\mu x} d x \\
& =\mu^{-v-1} e^{\beta \mu} \Gamma(v+1, \beta \mu), \text { Re } \mu>0 .
\end{aligned}
$$

And $\Psi_{3}$ in Eq. (36),

$$
\begin{aligned}
& \Psi_{3}=\Gamma\left\{m+1,\left(\frac{1+K}{\rho_{a}}+\frac{1}{\Gamma_{a} \rho_{g}}\right)\right\} \\
& \quad=m ! \exp \left\{-\left(\frac{(1+K) \Gamma_{a}}{\rho_{a}}+\frac{1}{\rho_{g}}\right)\right\} \sum_{q=0}^{m} \frac{1}{q !}\left(\frac{(1+K) \Gamma_{a}}{\rho_{a}}+\right. \\
& \left.\frac{1}{\rho_{g}}\right)^{q} .
\end{aligned}
$$

From Eq. (35), Eq. (36), and Eq. (38), the outage probability that UAM is a strong user can be obtained.

Consequently, the outage probability of UAM in the reverse link obtains by putting Eq. (20), Eq. (21), Eq. (33), and Eq. (34) into Eq. (32).

\subsection{Link balance}

The link quality balance between the forward link and the reverse link of UAM is important to provide stable voice calls for a passenger. To maintain the equal QoS between the two links, it is required that the outage probabilities have to be equal. The function of $\mathrm{Q}(\sqrt{2 K}, \sqrt{2(1+K) \chi})$ increases with $\chi$. Hence, when $\phi$ and $\varphi$ have equal values, the outage probabilities of the forward and reverse links are identical. From Eq. (24) and Eq. (31), this condition in weak AU can be written by

$$
\frac{\Gamma_{a}}{K_{1, w} \rho_{a}\left(\alpha_{w}-\Gamma_{a} \alpha_{s}\right)}=\frac{\Gamma_{a}}{\rho_{a}}, \quad\left|h_{a}\right|^{2} G_{a} \leq\left|h_{g}\right|^{2} G_{g}
$$

where $K_{1, w}=P_{b} / P_{a, w}, P_{a, w}$ is the transmit power of the $\mathrm{AU}$ in the weak user. From Eq. (39), we can rewrite

$$
P_{a, w}=P_{b}\left(\alpha_{w}-\Gamma_{a} \alpha_{s}\right), \quad\left|h_{a}\right|^{2} G_{a} \leq\left|h_{g}\right|^{2} G_{g} .
$$

Similarly, when the AU is a strong user, the link balance condition is given by

$$
\frac{\Gamma_{a}}{\alpha_{s} K_{1, s} \rho_{a}}=\frac{\Gamma_{a}\left(H_{g} \rho_{g}+1\right)}{\rho_{a}}, \quad\left|h_{a}\right|^{2} G_{a}>\left|h_{g}\right|^{2} G_{g} .
$$

where $K_{1, s}=P_{b} / P_{a, s}, P_{a, s}$ is the transmit power of the AU in the strong user. Since $H_{g}$ in Eq. (41) is the random variable, the average transmit power of the strong AU can be written by

$$
\begin{aligned}
P_{a, s} & =P_{b} \rho_{g} \alpha_{s} \int_{0}^{\infty} x e^{-x} d x+P_{b} \alpha_{s} \\
& =P_{b} \alpha_{s}\left(\rho_{g}+1\right),\left|h_{a}\right|^{2} G_{a}>\left|h_{g}\right|^{2} G_{g} .
\end{aligned}
$$

As shown in Eq. (40) and Eq. (42), the transmit power of the AU for link balance is changing, hence the BS transmit power to AU transmit power ratio of $K_{1, w}$ or $K_{1, s}$ are also changing. If Eq. (40) and Eq. (42) are same, it is the link balance condition irrespective of weak or strong AU. With that condition, the power allocation coefficient $\alpha_{w}$ becomes

$$
\alpha_{w}=\alpha_{s}\left(\rho_{g}+\Gamma_{a}+1\right)
$$

The average transmit power of the AU is given by

$$
\begin{aligned}
P_{a}= & P_{a, w} \operatorname{Pr}\left(\left|h_{a}\right|^{2} G_{a} \leq\left|h_{g}\right|^{2} G_{g}\right) \\
& +P_{a, s} \operatorname{Pr}\left(\left|h_{a}\right|^{2} G_{a}>\left|h_{g}\right|^{2} G_{g}\right) .
\end{aligned}
$$

And we can obtain Eq. (44) from Eq. (40), Eq. (42), Eq. (20) and Eq. (21).

\section{Numerical examples}

In this section, we discuss the probability of the strong or weak user of an AU. And the analytical and simulation results of outage probabilities of AU in the forward link and reverse link are compared. The simulation results are obtained from the Monte-Carlo

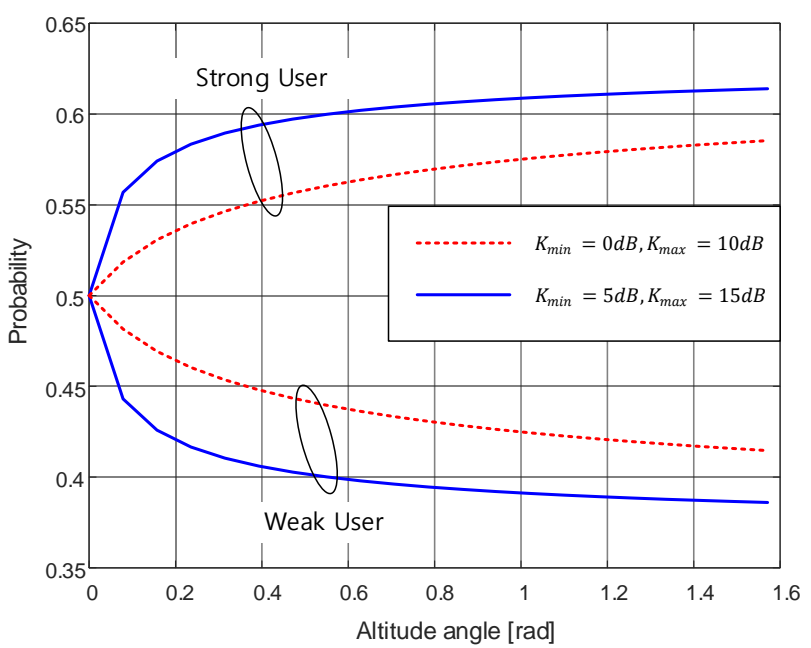

Figure. 2 Probability of strong/weak user 
simulation with $1 \times 10^{8}$ trials. Also, examples of the transmit power of the AU to maintain the link balance are addressed.

The probabilities of weak or strong user versus altitude angle are shown in Fig. 2. It is noticed that the increase of the altitude angle causes the increase of the LOS component, consequently, the probability of strong user increases.

Fig. 3 shows the outage probability of AU in the forward link, the sloid line and "*" denote the analytical and simulation results, respectively. We noticed that the analytical results coincide with the simulation results, which shows the analytical outage probability expressions have been validated. An increase of Rician $K$ factor incurs the increase of direct signal power and follows the decrease of the outage. Also, it is noticed that the consistent decrease of the outage probability with the increase of SNR.

The outage probability of $\mathrm{AU}$ in the reverse link is shown in Fig. 4, which shows the analytical results are exactly matched those obtained Monte-Carlo simulation. Different from the forward link, the outage probability does not decrease as the increase of the received SNR in the reverse link. It shows an error floor that has a flat outage probability above certain SNR. This is interpreted that the outage probability is affected by the interference of GU in the reverse link, however, isn't in the forward link. In the case of weak AU, the received signal removes the interference by SIC. However, the interference component still exists in the case of strong AU. Hence the outage probability, which is the average outage probability of weak and strong AU, is affected by the interference. Also, it shows the outage probability decreases as the increase of $K$, since the direct signal component increases.

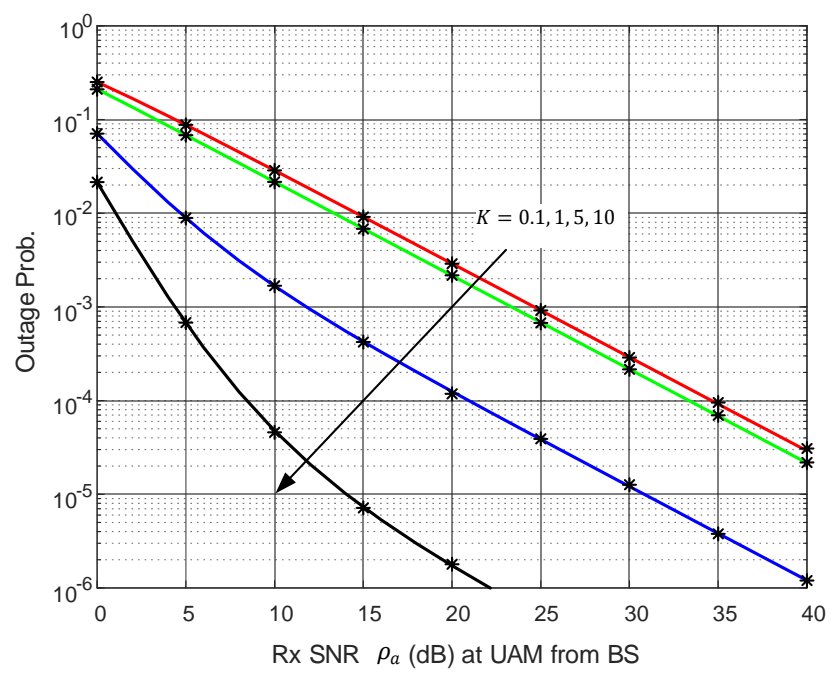

Figure. 3 Forward link outage probability $\left(R=1, \alpha_{w}=\right.$ $0.7, K 1=10 d B, G_{a} / G_{g}=1$ )

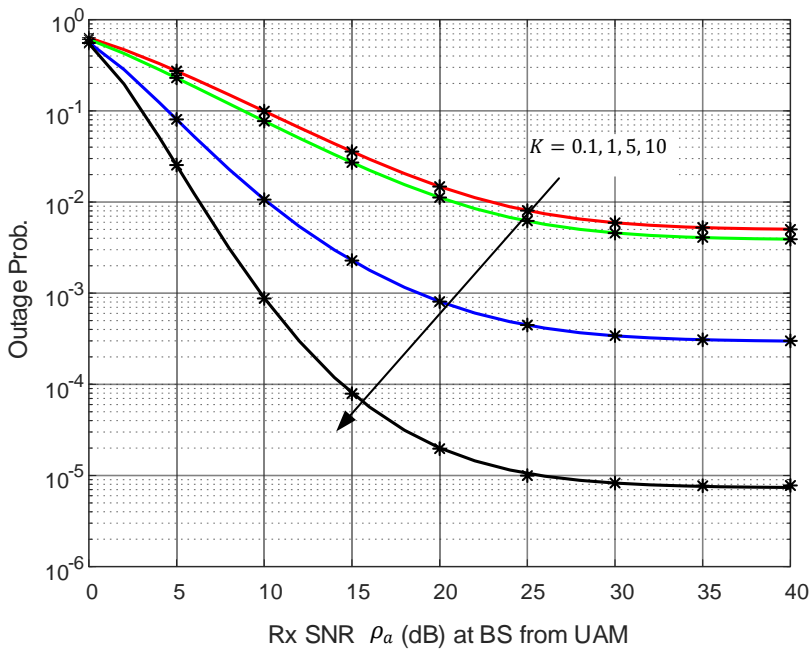

Figure. 4 Reverse link outage probability $(R=1, K 1=$ $\left.10 d B, \rho_{g}=\rho_{a}-20 d B, G_{a} / G_{g}=1\right)$

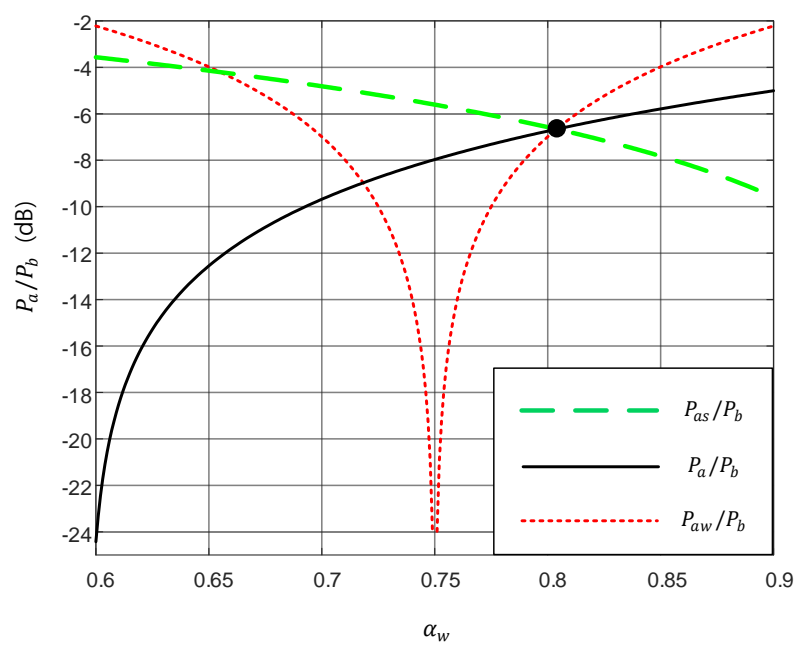

Figure. 5 UAM transmission power for link balance $(R=$ $\left.2, K=5, \rho_{a}=30 \mathrm{~dB}, \rho_{g}=\rho_{a}-40 \mathrm{~dB}, G_{a} / G_{g}=1\right)$

The condition of the link balance of an AU is shown in Fig. 5, where the curb of $P_{a s} / P_{b}$ and $P_{a w} / P_{b}$ denote the transmit power of strong AU and weak AU, respectively. The curb of $P_{a} / P_{b}$ denotes the average transmit power of the AU to maintain the link balance irrespective of strong or weak AU. In the case of strong $\mathrm{AU}, P_{a s} / P_{b}$ decreases with the power allocation coefficient $\alpha_{w}$, consequently, the outage probability also increases to satisfy the link balance. In the case of weak $\mathrm{AU}$, it is noticed that $P_{a w} / P_{b}$ curb decreases until $\alpha_{w}=0.75$, where $\alpha_{w}=\Gamma_{a} \alpha_{s}$ in Eq. (40), and later increases to satisfy the link balance. In this figure, the point of " $\bullet$ " denotes the AU to conform the link balance regardless of strong or weak user. At the point of $\alpha_{w}=0.804, P_{a s} / P_{b}, P_{a w} / P_{b}$, and $P_{a} / P_{b}$ equal $-6.66 \mathrm{~dB}$, we confirmed that the outage probabilities of forward and reverse links in Eq. (26) and Eq. (32), respectively, are identical to $1.33 \times$ 
$10^{-4}$. It means that we can afford identical QoS in forward and reverse links.

\section{Conclusions}

Deployment of UAM is actively discussed for air mobility in an urban area to avoid terrestrial traffic. Different from the conventional UAV, UAM is carrying cargo and passengers. Especially, the predetermined QoS must be satisfied for the voice traffic of passengers in forward and reverse links. This paper considered the UAM in NOMA cellular systems. We derived the outage probabilities of the UAM in forward and reverse links and conformed through the Monte-Carlo simulation. Also, the transmit power of UAM for the link balance, the outage probabilities of forward and reverse links are identical, was derived.

Numerical results showed that the probability of a strong user increased with the increase of altitude angle of UAM. The outage probability decreased with SNR in the forward link, however, the outage probability showed an error floor caused by the interference from a GU in the reverse link. The derived expressions exactly matched those obtained from the Monte-Carlo simulation. Also, we addressed the transmit power of UAM to satisfy the link balance.

Further research will be focused to find the minimum transmit power and minimum outage probability while satisfying the link balance.

\section{Conflicts of interest}

The authors declare no conflict of interest.

\section{References}

[1] Y. Saito, Y. Kishiyama, A. Benjebbour, T. Nakamura, A. Li, and K. Higuchi, "Nonorthogonal multiple access (NOMA) for cellular future radio access," In: Proc. of Vehicular Technology Conf. (VTC spring), Dresden, Germany, 2013.

DOI: 10.1109/VTCSpring.2013.6692652

[2] Z. Ding, Z. Yang, P. Fan, and H. V. Poor, "On the performance of non-orthogonal multiple access in 5G systems with randomly deployed users," IEEE Signal Processing Letters, Vol. 21, No. 12, pp. 1501-1505, 2014.

[3] X. Wang, J. Wang, L. He, and J. Song, "Outage analysis for downlink NOMA with statistical channel state information", IEEE Wireless Communication Letters, Vol. 7, No. 2, pp. 142145, 2018.
[4] Z. Yang, C. Pan, W. Xu, Y. Pan, M. Chen, and M. Elkashlan, "Power control for multi-cell networks with non-orthogonal multiple access", IEEE Transactions on Wireless Communications, Vol. 17, No. 2, pp. 927-942, 2017.

[5] Z. Dong, M. Peng, and H. V. Poor, "Cooperative non-orthogonal multiple access in $5 \mathrm{G}$ systems", IEEE Communications Letters, Vol. 19, No. 8, pp. 1462-1465, 2015.

[6] X. Liang, Y. Wu, D. W. K. Ng, Y. Zuo, S. Jin, and $\mathrm{H}$. Zhu, "Outage performance for cooperative NOMA transmissions with an AF relay", IEEE Communications Letters, Vol. 21, No. 11, pp. 2428-2431, 2017.

[7] Z. Ding, F. Adachi, and H. V. Poor, "The application of MIMO to non-orthogonal multiple access", IEEE Transactions on Wireless Communications, Vol. 15, No. 1, pp. 537-552, 2016.

[8] Q. Sun, S. Han, C. L. I, and Z. Pan, "On the ergodic capacity of MIMO NOMA systems", IEEE Wireless Communication Letters, Vol. 4, No. 4, pp. 405-408, 2015.

[9] F. Zhou, Y. Wu, Y. C. Liang, Z. Li, Y. Wang, and K. K. Wong, "State of art, taxonomy, and open issues on cognitive radio networks with NOMA", IEEE Wireless Communications, Vol. 25, No. 2, pp. 100-108, 2018.

[10] N. S. Kim, "Overlay cognitive radio NOMA networks with selective relay and direct link", International Journal of Intelligent Engineering and Systems, Vol. 13, No. 1, pp. 181-190, 2020.

[11] N. S. Kim, "Cooperative overlay cognitive radio NOMA network with channel errors and imperfect SIC", International Journal of Intelligent Engineering and Systems, Vol. 12, No. 5, pp. 224-231, 2019.

[12] M. Monemi, H. Tabassum, and R. Zahedi, "On the performance of non-orthogonal multiple access (NOMA): Terrestrial vs. aerial networks", In: Proc. of ComNet2020, Hammamet, Tunisia, pp. 1-8, 2020.

[13] A. Han, T. Lv, and X. Zhang, "Outage performance of NOMA-based UAV-assisted communication with imperfect SIC", In: Proc. of Wireless Communications and Networking Conf. (WCNC), Marrakesh, Morocco, pp. 1-6, 2019.

[14] C. Guo, C. Guo, S. Zhang, and Z. Ding, "UAVenabled networks analysis with selective incremental relaying and imperfect CSI", IEEE Transactions on Vehicular Technology, Vol. 69, No. 12, pp. 16276-16281, 2020. 
[15] M. M. Azari, F. Rosas, K. C. Chen, and S. Pollin, "Ultra reliable UAV communication using altitude and cooperation diversity", IEEE Transactions on Communications, Vol. 66, No. 1, pp. 330-344, 2018.

[16] W. K. New, C. Y. Leow, K. Navaie, and Z. Ding, "Robust non-orthogonal multiple access for aerial and ground users", IEEE Transactions on Wireless Communications, Vol. 19, No. 7, pp. 4793-4805, 2020.

[17] S. K. Mahmud, Y. Liu, Y. Chen, and K. K. Chai, "Adaptive reinforcement learning framework for NOMA-UAV networks", IEEE Communications Letters, Vol. 25, No. 9, pp. 2943-2947, 2021.

[18] X. Yang, L. Deng, J. Liu, P. Wei, and H. Li, "Multi-agent autonomous operations in urban air mobility with communication constraints", In: Proc. of American Institute of Aeronautics and Astronautics (AIAA) Scitech Forum, Orlando, FL, pp. 1-12, 2020.

[19] M. K. Simon and M. S Alouini, Digital communication over fading channels, John Wiley\&Sons, New York, N.Y. 2000.

[20] M. Mozaffari, W. Saad, M. Bennis, and M. Debbah, "Unmanned aerial vehicle with underlaid device-to-device communications: on performance and tradeoffs", IEEE Transactions Wireless Communications, Vol. 15, No. 6, pp. 3949-3963, 2016.

[21] A. Annamalai, C. Tellambura, and J. Matyjas, "A new twist on the generalized Marcum Qfunction $Q_{M}(a, b)$ with fractional-order $\mathrm{M}$ and its applications", In: Proc. of IEEE Consumer Communications and Networking Conf., Las Vegas, NV, pp. 1-5, 2009.

[22] I. S. Gradshteyn and I. M. Ryzbik, Table of integrals, series, and products, $6^{\text {th }}$ Ed., Academic Press, London, UK, 2002. 